\title{
Materials behaviour and numerical simulation of a turbine blade-off containment analysis
}

\author{
F. Gálvez, D. A. Cendón, A. Enfedaque \& V. Sánchez-Galvez \\ Dpto. Ciencia de Materiales, E.T.S.I. Caminos Canales y Puertos, \\ Universidad Politécnica de Madrid, Spain
}

\begin{abstract}
This paper analyses the impact phenomenon of a failed blade against the containment inside a turbine (called "blade off") by using the Finite Element Method. In addition, the research has also focused on the secondary damage, which is the damage induced by the debris of the lost blade on the adjacent blades of the turbine. The paper shows how the model has been arranged from the beginning, pointing out aspects concerning the materials, initial and boundary conditions. The study shows the importance of having well known characterization of the materials involved, including the effects of high strain rate and high temperature. The results show how both blade off and secondary damage phenomena can be accurately modelled by FEM and provides useful information for the entire process.
\end{abstract}

Keywords: high strain rate, impact, numerical simulation.

\section{Introduction}

In the rare but possible event of a turbine blade or fan blade failure, the engine must ensure the containment of it. In other case the blade becomes a projectile which can seriously damage the aircraft, causing occupants injuries and eventually leading the airplane to crash. Because this reason, the fan and turbine containments are usually the heaviest parts of the engines and during the engine design process, the containment must somehow be ensured. Up to date, the containment was designed by using certain kind of abacuses from some experimental tests and more or less documented blade failure real cases. Despite this can be a quite sure designing tool, it also has some disadvantages: for example it is not possible to know which would be the critical thickness of the 
containment (that is to say, which is the safety factor), or if an engine designer wants to design a new type of blade maybe the abacuses are not applicable because of the shape of the blade, and performing a real containment test is always very expensive and time consuming.

Because these reasons, numerical hydrocodes are increasingly being used to the blade containment analysis. The input parameters are usually the following: geometry of the blades and containment, material properties (sometimes not fully characterized for those temperature and strain rate ranges), operating temperature profile and maximum engine turning speed.

In this particular case, besides analysing the containment, it was desired to analyze the secondary damage, which is the damage caused by the debris of the blade which has failed and impacted against the containment on the trailing blades which still turns fixed to the turbine.

\section{Problem description}

A turbo jet engine stage basically consists on a group of blades fitted in a disk. Under operating conditions, the disk-blades set rotate around the disk axis with a high speed. Because some crack, material defect etc, one blade (named lost blade) could fail and it would be launched against the containment. To simulate this situation, only one blade and the containment have to be modelled. However, as we also want to analyse the secondary damage, we need to model at least one blade adjacent to the lost one.

Figure 1a shows the geometry of a blade with some important parts of it. Figure $1 \mathrm{~b}$ shows the initial position of two blades inside the containment. During the failure process it will be supposed that the engine turns with a constant speed and that the failure arises suddenly, that is to say: the crack appears instantaneously.

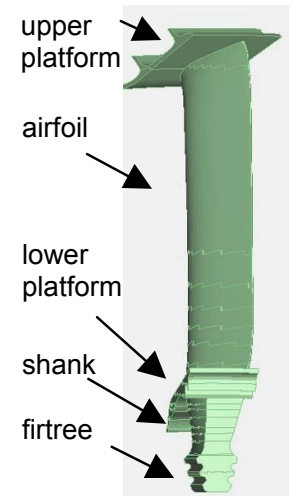

a)

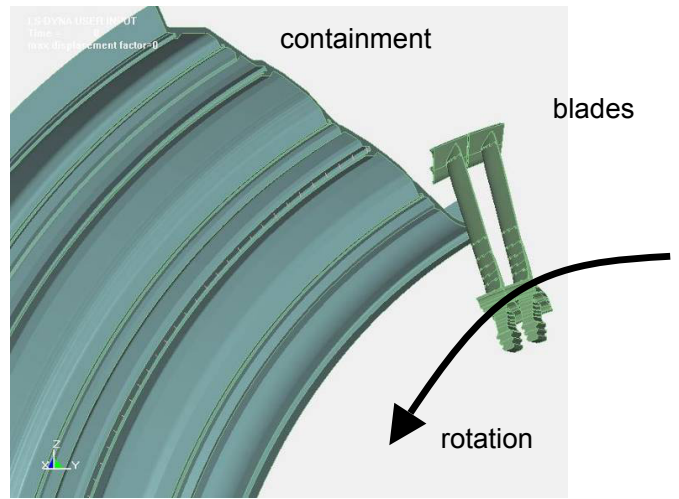

b)

Figure 1: a) Blade geometry and parts of it; b) Initial position of blades and containment. 


\section{Material models}

To use correct constitutive models for both the containment and the blade materials, it is necessary to understand the operating conditions during the event. The temperature of the case material is in the $400-600^{\circ} \mathrm{C}$ range, while the temperature of the blades is in the $800-900^{\circ} \mathrm{C}$ range. Additionally, as the impact of the blade against the case is studied, the strain rates could be high and the strain rate sensitivity is also needed for both materials.

The model selected for the case material (steel FV535) was the Johnson-Cook [1] material model. To obtain the parameters of this model, an experimental campaign of mechanical tests was developed. To characterize the material at low strain rates several compression tests on cylinders were performed at strain rates of $10^{-3} \mathrm{~s}^{-1}$ and temperatures ranging from 24 to $850^{\circ} \mathrm{C}$. To obtain the behaviour at high strain rates, Hopkinson bar tests were also performed at the same temperature range and obtaining strain rates of $10^{3} \mathrm{~s}^{-1}$. The utilization of the Hopkinson bar technique with a furnace for this purpose has been previously studied and proposed by the authors [2]. The parameters of the $\mathrm{J}-\mathrm{C}$ material model were obtained looking for the best fit within the $400-700^{\circ} \mathrm{C}$ range, which fits with the temperature interval where this material is used on the application studied here. The parameters obtained for this model are shown in Figure 2, also showing the yield stress obtained from the static and dynamic tests as well as the J-C fit. As it can be observed, the model reproduces well the material behaviour, even better on the $400-800^{\circ} \mathrm{C}$ range.

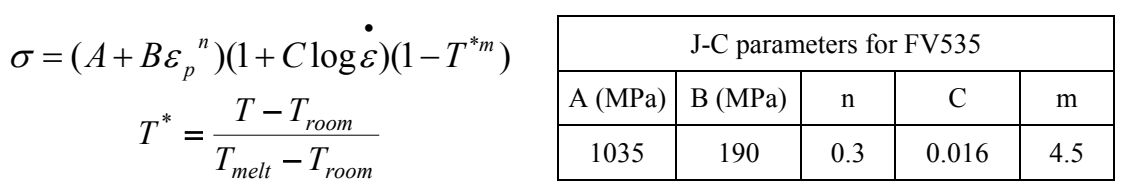

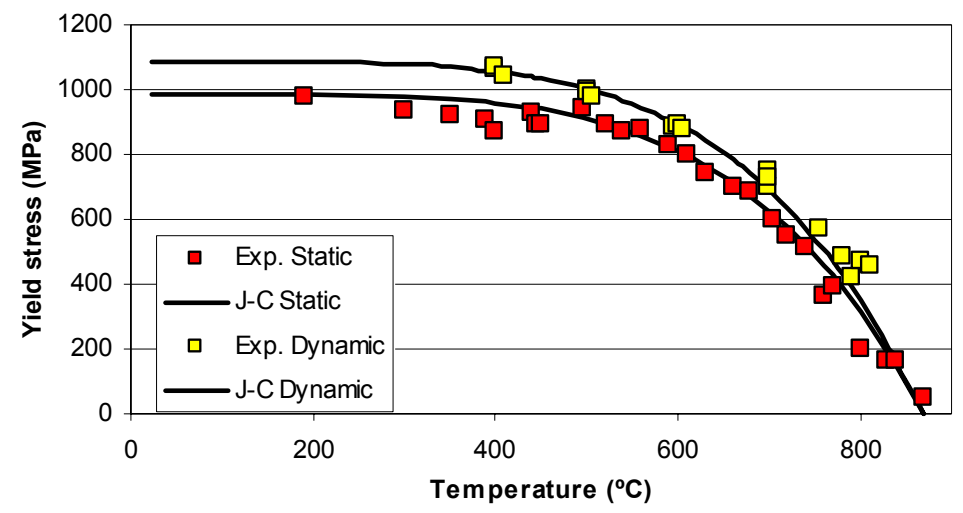

Figure 2: Yield strength and its Johnson-Cook fit for the material of the containment. 
For the blade material, our first idea was also to use a Johnson-Cook strength model. However it was not possible to make an experimental testing campaign to obtain the materials behaviour. As an alternative, the engine manufacturer provided us the material stress-strain curves with a potential fitted law for different temperatures. Those curves represent the plastic region of the material. With a careful look of the material data, we observed that the material response from $20^{\circ} \mathrm{C}$ to $800^{\circ} \mathrm{C}$ could be considered constant and the temperature effect in this range could be considered insignificant to our purposes. So we decided to use a custom version of the Johnson-Cook model to represent well the material behaviour. This model is represented with a similar function than $\mathrm{J}-\mathrm{C}$ but using true strains in stead of plastic strains (Figure 3). To include the strain rate effects as well as the temperature effects, similar laws than J-C were used. To compute the stresses (Figure 3), the model checks if the elastic stress computed versus the potential law. If less, the material remains elastic and, but if greater the material has reached plasticity and the potential law is used. We also decided to not include the behaviour below $800^{\circ} \mathrm{C}$ but take into account the temperature effect from $800^{\circ} \mathrm{C}$ and up. To do it, the model checks the temperature on each element is less than $800^{\circ} \mathrm{C}$. If it is, a temperature of $800^{\circ} \mathrm{C}$ is assigned to the element, if not it remains unaltered. To include also the strain rate effect, as no data was available, we decided to use the same strain rate effect obtained by other authors using Johnson-Cook models [3], by including the same type of logarithmic dependence with similar C constant.

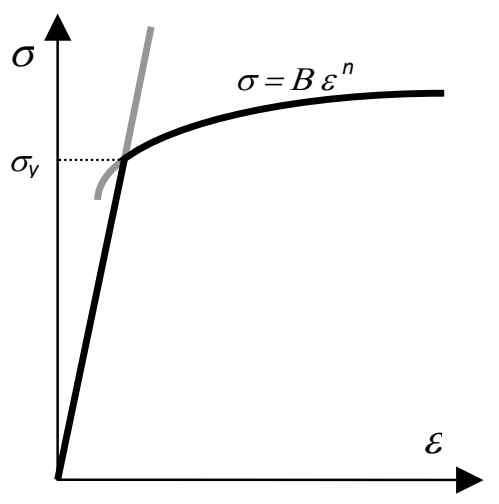

$$
\begin{gathered}
\sigma=B \varepsilon^{n}(1+C \log \dot{\varepsilon})\left(1-T^{* m}\right) \\
T^{*}=\frac{T_{e f}-T_{\text {room }}}{T_{\text {melt }}-T_{\text {room }}} \\
\text { if } T<T_{\text {room }} \text { then } T_{\text {ef }}=T_{\text {room }} \\
\text { if } T \geq T_{\text {room }} \text { then } T_{\text {ef }}=T
\end{gathered}
$$

\begin{tabular}{|c|c|c|c|c|}
\hline \multicolumn{5}{|c|}{ Material model parameters for IN718 } \\
\hline $\begin{array}{c}\mathrm{B} \\
(\mathrm{MPa})\end{array}$ & $\mathrm{n}$ & $\mathrm{C}$ & $\mathrm{m}$ & $\begin{array}{c}T_{\text {room }} \\
\left({ }^{\circ} \mathrm{C}\right)\end{array}$ \\
\hline 1375 & 0.085 & 0.017 & 1.15 & 800 \\
\hline
\end{tabular}

Figure 3: $\quad$ Material model description for the IN718Alloy.

The failure criterion selected for the case material was based on the maximum principal strain because of its simplicity and because the experimental data did not provide failure (tests were based on compression configuration). In the case of the blades, the Johnson-Cook failure model [4] was selected. Unfortunately since this failure criterion has many parameters, a large variety of tests must be conducted in order to measure such parameters: different strain rates, temperatures, combinations of stresses, etc, and so if it is difficult to have 
parameters to properly fit the yielding stress of the Johnson-Cook strength model, much more difficult is to have data enough to fit the Johnson-Cook failure criterion. In the case of the blades, the Johnson-Cook failure model [4] was fitted ensuring that for the direct tensile test conditions the maximum strain were equal to that provided by the manufacturer, and under direct compressive test conditions the maximum strain were as high as possible, to avoid element erosion on the initial stages of the blade impact against the containment, when the compressive stresses are very high, but the failure of the material under these conditions has no physical meaning. Some researchers have reported this problem using this failure criterion in some impact numerical simulations with hydrocodes [5].

\section{Boundary and initial conditions}

To avoid an excessively large number of degrees of freedom, only one quarter of the containment was modelled. To prevent wave reflection, non-reflection boundary conditions were prescribed in all free edges (Figure 4a). In the vertical bridles of the containment, displacement parallel to the engine axis was fixed to zero (Figure 4b). Regarding the blades, in the outer nodes of the trailing blades socket (firtree), besides a prescribed constant velocity rotation around the axis, the displacement parallel to it was also fixed to zero (Figure 4c). The initial conditions of the model were the operating temperature profile, and the initial velocity of the blades.

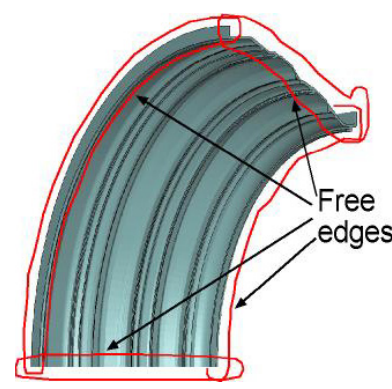

a)

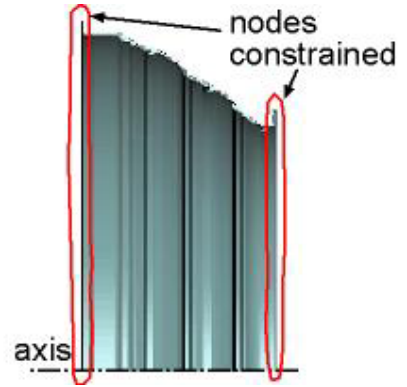

b)

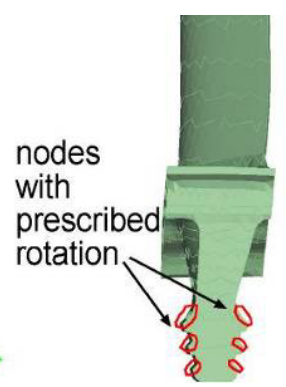

c)

Figure 4: a) Free edges of the containment with non-reflection boundary conditions; b) Node sets with parallel-to-axis displacement prescribed to zero; c) Blade nodes prescribed to rotate.

In the aim of reduce the maximum degree of freedom number, initially only two blades and the containment were modelled, as displayed in Figure 1. For the analysis it was supposed that the blade suddenly fails, that is to say, no progressive fracture is taken into account and the simulation starts with the shank blade completely broken. Following classical mechanics, once the shank is broken no forces can be applied to the lost blade and then, it must keep its straight and constant movement; in other words: the blade is ejected against the 
containment following a tangential trajectory (Figure 5a). However, under operating conditions the blades are not isolated, and the lower platform of one blade is almost in contact with the lower platforms of the neighbour blades. This constraint makes impossible the ejection of the blade following a tangential trajectory because the blade must first to free from their neighbour blades following a normal trajectory (Figure 5b). Once the blade has got it, it is free to follow the tangential trajectory, but it also has a normal velocity what substantially changes the initial conditions of only tangential velocity.

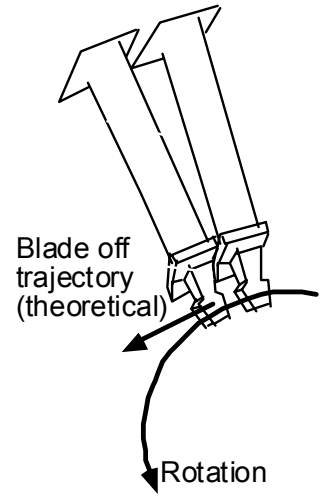

a)

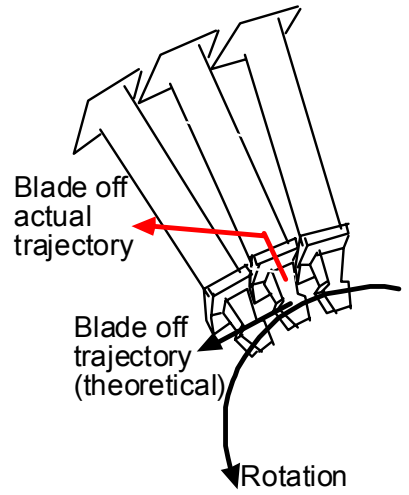

b)

Figure 5: a) Theoretical lost blade trajectory; b) Actual lost blade trajectory.

These initial velocity conditions can be obtained, either starting the simulation when the blade has been broken but still at is original position, or obtaining the initial velocity after the blade has got free from its neighbours using a physics based simplification. In the preliminary calculations, this simplification was used. This method allows to reduce the time length of simulation (since the blade ejection process is not modelled) and the number of degrees of freedom, since only requires to model the lost blade and the adjacent trailing blade.

However, once the lost blade has stroked the containment, the trailing blade impacts against it, suffering a large airfoil deflection (Figure 6a). In an actual turbine, this large deflection can never happen, since it would be impeded by the second adjacent blade (Figure $6 \mathrm{~b}$ ). To take this into account on the simulations, a third blade was included, and also the platforms of two additional blades. With such improvement, the number of degrees of freedom was increased in such way that the cost reduced improvement obtained with the physics based simplification was irrelevant so it was finally skipped, starting the simulations with the shank broken and the blade at its initial position.

\section{Initial stresses}

If the model is run prescribing only the initial velocities and boundary conditions, it can be appreciated that some regions of the trailing blade fail, or 
are close to fail, before any impact in the model is observed. Furthermore, analysing the stress contours of the blades, a wide oscillation can be observed ranging from zero to values that in some cases causes material to go into plastic regime, something surprising having in mind that the trailing blade is still under normal operating conditions. The reason for such strange behaviour can be found in the initial stresses: despite having initialized the velocity of the blades to the operating ones, the simulation starts with no stresses, what is not in equilibrium with the initial turning velocity of the blades. This causes wave propagation like in a non elongated spring trying to equilibrate a moving mass.

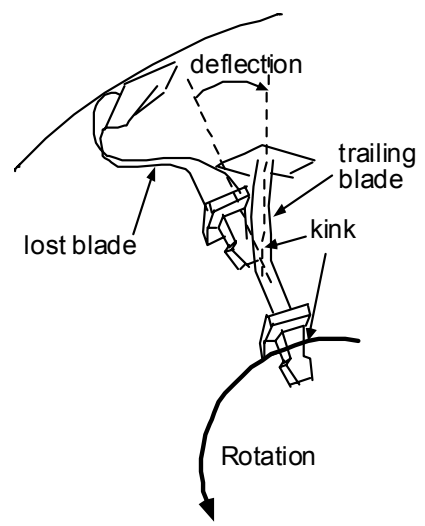

a)

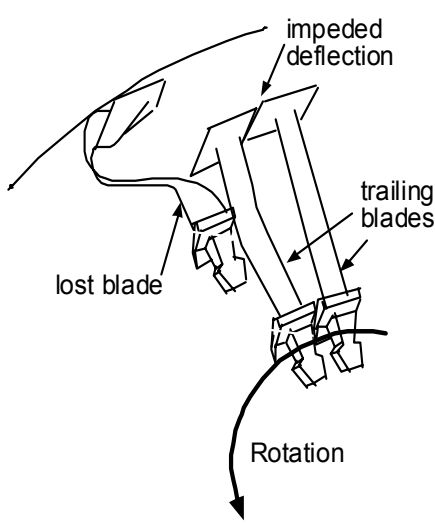

b)

Figure 6: a) Sketch showing the blade deflection if only one trailing blade is modelled; b) Id. if more than one trailing blade is modelled.

To avoid this stress oscillation, it is necessary to initialize the stresses in the blade with the values that equilibrates the initial turning of them around the engine axis with a constant turning speed. For that purpose an initial static calculation was performed, applying the acceleration field of a constant velocity rotation to the blades. The resulting stress field was prescribed as an initial condition to the blades in the explicit calculation.

The Von Mises stress history measured at the same element of the mesh, with and without the stresses initialization before any impact at the trailing blade is shown in Figure 7. The difference is obvious.

\section{Element types}

For the containment, due to its cylindrical symmetry, it was easy to achieve a hexahedrical element mesh, meshing a cross section by quadrilateral plane elements and then extruding it turning through the symmetry axis.

Regarding the blade, it is well known that the use of tetrahedrons in the mesh usually leads to worse results than if hexahedrons are used. In plasticity problems tetrahedrons lead to more stiff behaviour due to their limitations 
tracing uncompressible modes of strain. However, they still are widely used, especially when complex pieces and shapes are present in the simulations. This is mainly because up to date a fully automatic hexahedrical mesher has not been developed yet. The blades present in this problem were very difficult to mesh using hexahedrons and because this reason tetrahedrons were used in the first calculations.

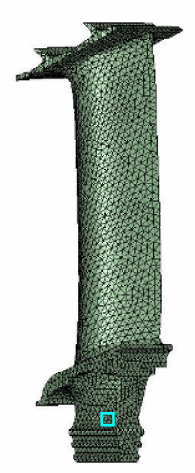

Element analyzed

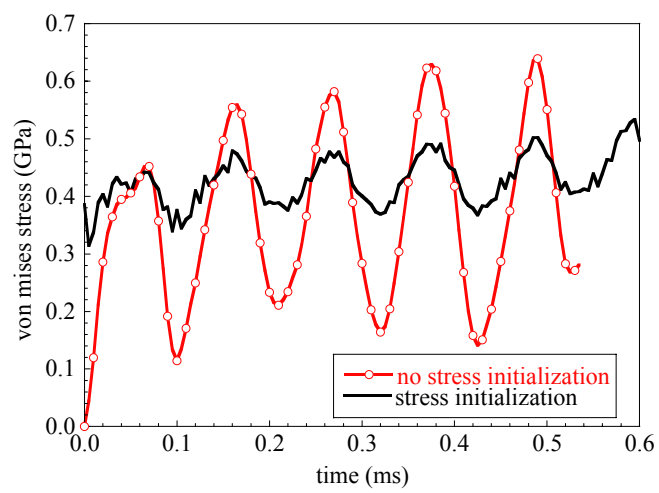

Figure 7: Von Mises stress history with and without stress initialization.

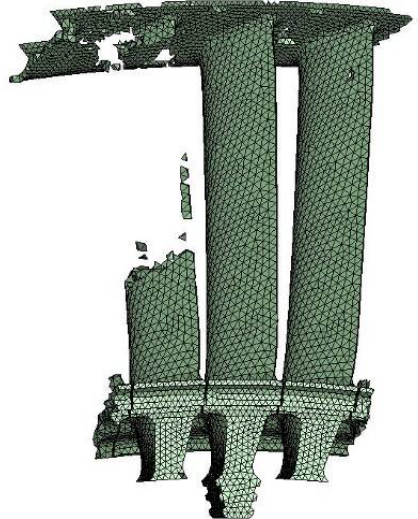

a)

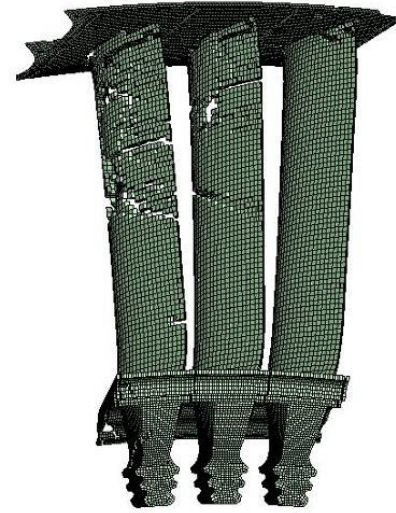

b)

Figure 8: Failure patterns, a) using tetrahedrons based mesh; b) using hexahedrons based mesh.

Figure 8a shows the failure patterns of the blades after the simulation using tetrahedrons. It strongly calls the attention the large number of elements eroded on the failing blade. Looking to all the impact process, it can be observed that the behaviour of that blade is much more brittle than it should be having in mind that it is a metallic material with a maximum strain greater than a $10 \%$. The reason for such brittle behaviour can be found on the use of constant stress tetrahedrons: 
they behave too rigid to trace large plastic strains. This phenomenon is worsened by the fact that in the airfoil mesh there is only one tetrahedron through the thickness.

For that reason, although complicated, a hexahedrons based mesh was performed for the blades. As above mentioned, the shape of the blades is very complex and so, the entire blade was divided into three different pieces easier to mesh with hexahedrons. The three resultant meshed pieces were coupled to behave as a single piece using contact definitions [5]. This technique, although being not as accurate as a single mesh, simplifies notably the meshing process and provides reasonable results. In Figure $8 \mathrm{~b}$ can be observed the failure patterns and so far they look much more realistic than those obtained by using tetrahedrons.

\section{Results}

The first result is obtained analyzing the differences between the simulation using tetrahedrons and hexahedrons. The failure patterns (Figure 8) are completely different on both simulations, as well as the internal energy absorbed by the containment (Figure 9). As above mentioned, it is well known that tetrahedrons provide less accurate results than bricks. However, comparing both results, we cannot talk about lack of accuracy because results are completely different. Despite we do not have experimental results, the failure patterns obtained with the tetrahedrons suggest that the results obtained with this mesh are not only inaccurate but they seem to be completely wrong. The conclusion then is that sometimes the use of tetrahedrons are not useful neither to perform a rough simulation.

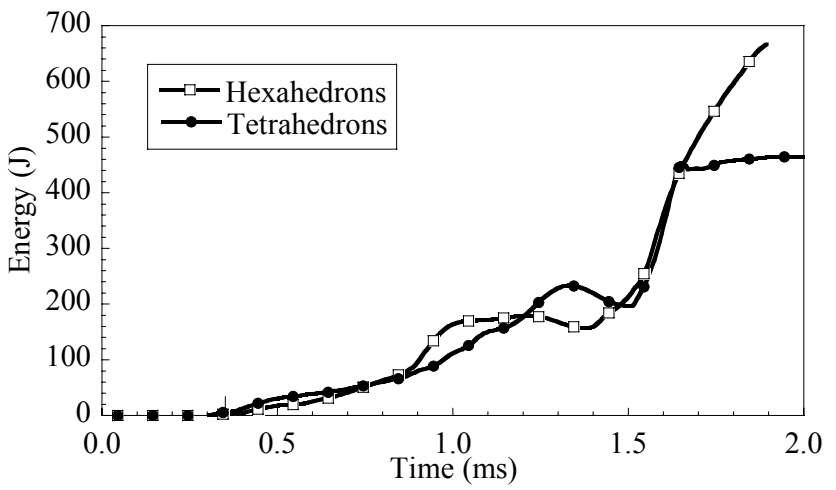

Figure 9: Containment internal energy history with both hexahedrons and tetrahedrons based meshes.

On other hand, it calls strongly the attention that the worst situation for the containment is not the initial strike of the lost blade against it, but the secondary strike of the blade debris against the containment after being pushed by the 
trailing blades. Figure 10 shows a sequence of the process at different simulation times. It also shows the history of internal energy absorbed by the containment. It can be appreciated how the trailing blade impacts against the debris of the failed blade, causing it to rotate and to strike again the containment, what produces a large amount of absorbed internal energy in the containment. This result is very important, since in order to study the blade off containment, many tests have been conduced based on shooting a blade against a piece of containment. Although being a useful test in order to calibrate material parameters, it does not reproduce what really happens inside the turbine, so it should not be used, for example, for containment dimensioning.

It is also important the influence of the gap between blade platforms. In Figure 11, it can be observed how the first trailing blade also fails and is ripped from its original position. It is very important that debris produced by the secondary damage must not produce a large amount of material, because they will strike against the following trailing blades, causing them again to fail. The larger amount of material the more possibilities for the containment failure. In this simulation it can be observed how the first trailing blade has failed through the airfoil, which produces much less amount of debris than if the failure had taken place through the shank (below the lower platform). If the gap between blades would have been greater, then the blade would have fail through the shank, because under operating conditions is the more stressed part of the blade and with a large gap the strike against the lost blade would have produce a large kink in the shank, inducing high stress values. This fact is important from the point of view of the simulations and from the point of view of turbines design.

\section{Conclusions}

The use of tetrahedrons must be avoided as far as possible. The large plastic strains developed during an impact phenomenon cannot be traced by this type of element. This leads, not only to a stiffer material behaviour, but also to a very different structural behaviour and a very different result when solving complex problems.

The rotation induces initial stresses in some kind of projectiles, such as the blade analyzed in this paper. Those stresses must be calculated and taken into account in the models if we want to obtain consistent results. In the case of this blade, the non inclusion of this stresses would lead to material plasticity in the blades under operating conditions which is against static analyses used for blade dimensioning.

Focusing on the blade impact analysis, it has been proved that the initial strike of the blade against the containment is not the worst consequence of the blade off: the blade debris impact trailed by the other blades of the turbine produces much more damage in the containment.

The blade-off phenomenon, as well as the secondary damage, has been successfully modelled using a commercial numerical code, providing a new tool for blade and containment design avoiding the need of full scale test, very complicated and expensive. 
a)

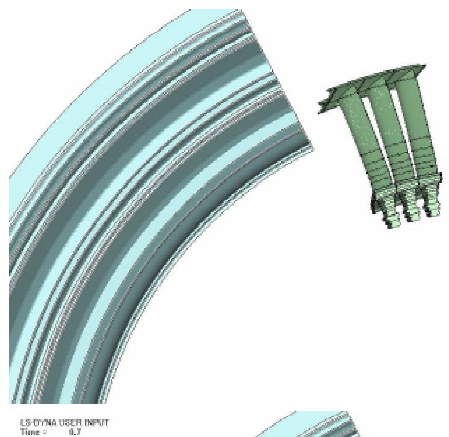

c)

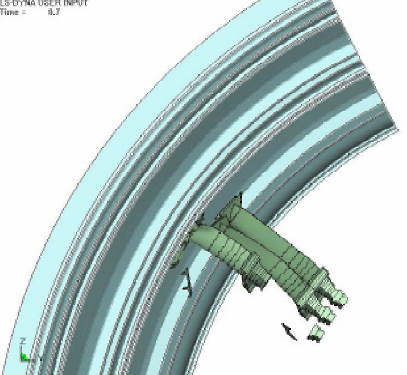

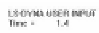

e)

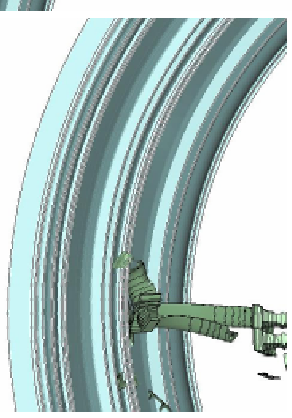

b)

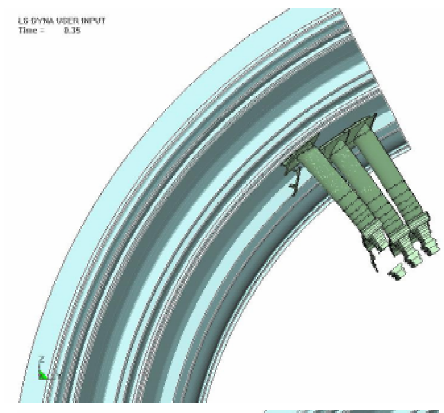

d)
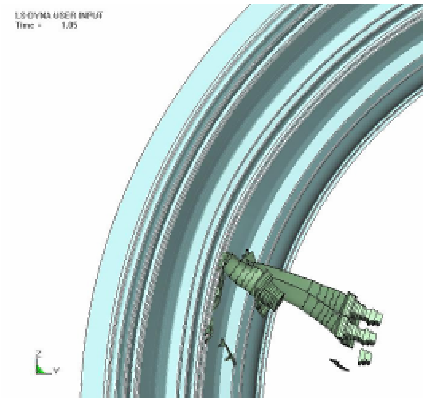

f)

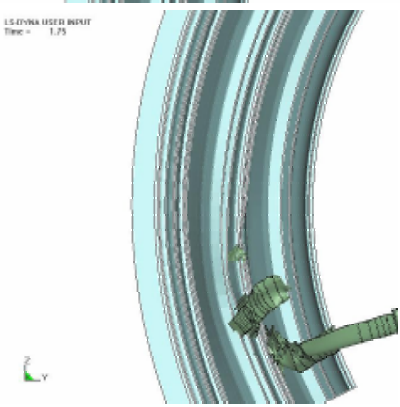

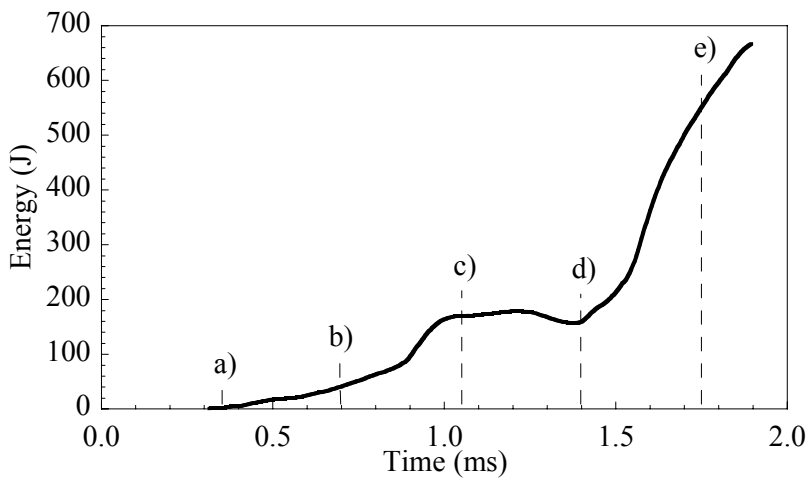

Figure 10: Sequence of the blade of simulation correlated with the containment internal energy history.

WIT Transactions on The Built Environment, Vol 87, (C) 2006 WIT Press www.witpress.com, ISSN 1743-3509 (on-line) 


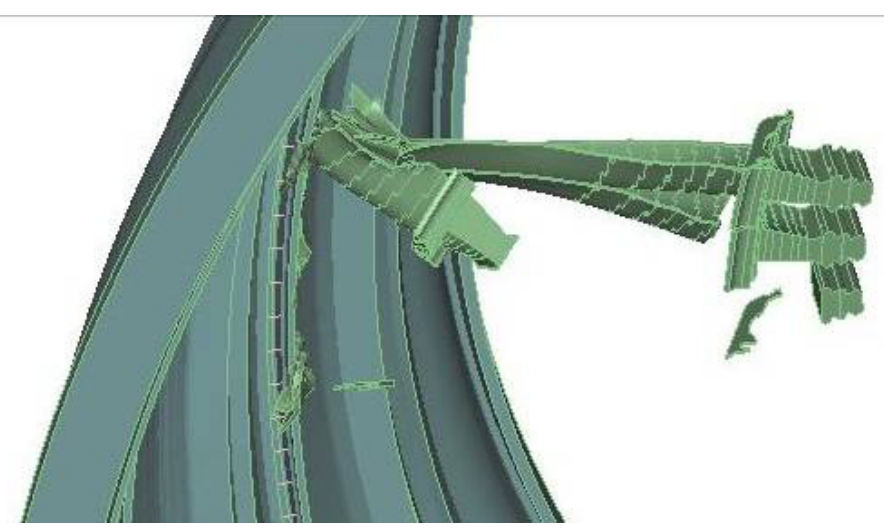

Figure 11: The trailing blade is also ripped, failing on the airfoil during the simulation.

\section{References}

[1] Johnson, G.R. \& Cook, W.H. A constitutive model and data for metals subjected to large strains, high strain rates and high temperatures. Proc. Seventh Int. Nat. Symposium on Ballistics, 1983.

[2] Gálvez F., Cendón D., Sánchez-Gálvez, V., Ensayos mecánicos en materiales a elevada velocidad de deformación y alta temperature Anales de Mecánica de la Fractura, 22, pp. 508-513, 2005.

[3] Pereira, J.M., Lerch, B.A, Effects on heat treatment on ballistic impact properties of Inconel 718 for jet engine fan containment applications Int, J, of Impact Engng. 25 (2001) 715-733.

[4] Singh, K.N., Clos, R., Schreppel, U., Veit, P., Hamann, A., Klingbeil, D., Sievert, R. and Künecke, G. Versagenssimulation dynamisch belasteter Proben mit unterschiedlichen Mehrachsigkeitszuständen unter Verwendung des Johnson-Cook-Versagensmodells für eine Nickelbasislegierun. Proc. Technische Mechanik. Pp 205-215. 2003.

[5] Carney, K., Pereira, M., Revilock, D and Matheny, P. Jet engine fan blade containment using two alternate geometries. 4th European LS-DYNA Users Conference, 2003.

[6] LS DYNA 3D Version 960. User Manual. Livermore Software Technology Corporation. 1998. 